\title{
Delocalized cationic azo dyes derived from 2-aminoselenazole-5-carbaldehyde
}

\author{
Maria A. Salvador, ${ }^{a}$ Lucinda V. Reis, ${ }^{a}$ P. Almeida, ${ }^{b}$ and Paulo F. Santos ${ }^{\mathrm{a}}$ * \\ ${ }^{a}$ Departamento de Química and Centro de Química - Vila Real, \\ Universidade de Trás-os-Montes e Alto Douro, Apartado 1013, 5001-801 Vila Real, Portugal \\ ${ }^{\mathrm{b}}$ Departamento de Química and Unidade de I \& D de Materiais Têxteis e Papeleiros, \\ Universidade da Beira Interior, 6201-001 Covilhã, Portugal \\ E-mail:psantos@utad.pt
}

\begin{abstract}
Several delocalized cationic azo dyes incorporating a selenazole ring have been prepared by Knoevenagel condensation of an intermediate azo compound bearing a 5-formylselenazole group with methylene bases derived from indolenine, benzothiazole, benzoselenazole, and quinoline. All dyes display a strong absorption at around $700 \mathrm{~nm}$, bathochromically shifted relative to their thiazole analogues, and show a negative solvatochromic behavior.
\end{abstract}

Keywords: Azo dyes, delocalized, cationic, selenazole, solvatochromism

\section{Introduction}

More than half of all commercially available dyes are azo dyes. ${ }^{1}$ Only a few azo dyes absorb in the near infrared (NIR), ${ }^{2}$ and among these, delocalized cationic dyes are rare. ${ }^{2 f}$ The majority of NIR azo dyes display bathochromism of the thiazole ring ${ }^{3}$ incorporated in the chromophore.

Recently, we have reported the synthesis of some delocalized cationic azo dyes derived from 2-amino- and 2-amino-4-chlorothiazole-5-carbaldehyde ${ }^{2 \mathrm{~g}, \mathrm{~h}}$ with absorption in the longwavelength region of the visible spectrum. Delocalized cationic azo dyes are a borderline case of polymethine dyes. ${ }^{4}$ We anticipated that the substitution of sulfur by selenium would have a bathochromic effect as observed with methine dyes derived from $N, N$-disubstituted 2aminothiazoles and 2-aminoselenazoles. ${ }^{5}$ Therefore, we synthesized several representative examples of delocalized cationic azo dyes based on Knoevenagel condensation of an azo derivative 5 of 2-aminoselenazole-5-carbaldehyde (3) with heterocyclic methylene bases. 


\section{Results and Discussion}

The synthetic strategy used to prepare the dyes described herein, i.e., the incorporation of a selenazole moiety into the chromophore, parallels that previously reported for their thiazole analogues. ${ }^{2 g}$ The selenazole features a diazotable amino group at C-2 to be coupled to an aromatic amine, and a formyl group at C-5, suitable to undergo Knoevenagel condensation with a cationic methylheterocycle.

Whereas several syntheses have been described for 2-aminothiazole-5-carbaldehyde, ${ }^{6}$ the selenium analogue appears to be unknown. The synthesis of 2-aminoselenazole-5-carbaldehyde (3) was accomplished in rather good yield by a modified Hantzsch method from bromomalonaldehyde (1) and selenourea (2) (Scheme 1). It is worthwhile mentioning that despite the desired structural diversity of 1,3 -selenazoles due to the growing interest in their potential biological activities, ${ }^{7}$ only few syntheses of 5-acyl-2-amino-1,3-selenazoles have been reported. ${ }^{8}$ 2-Aminoselenazole-5-carbaldehyde (3) was subsequently diazotized with nitrosyl bisulfate in DMF, and the resulting diazonium salt $\mathbf{4}$ was coupled with $N, N$-diethylaniline to afford the azo dye 5 in 17\% yield. Although different diazotization/coupling conditions were used, we were unable to improve the yield.
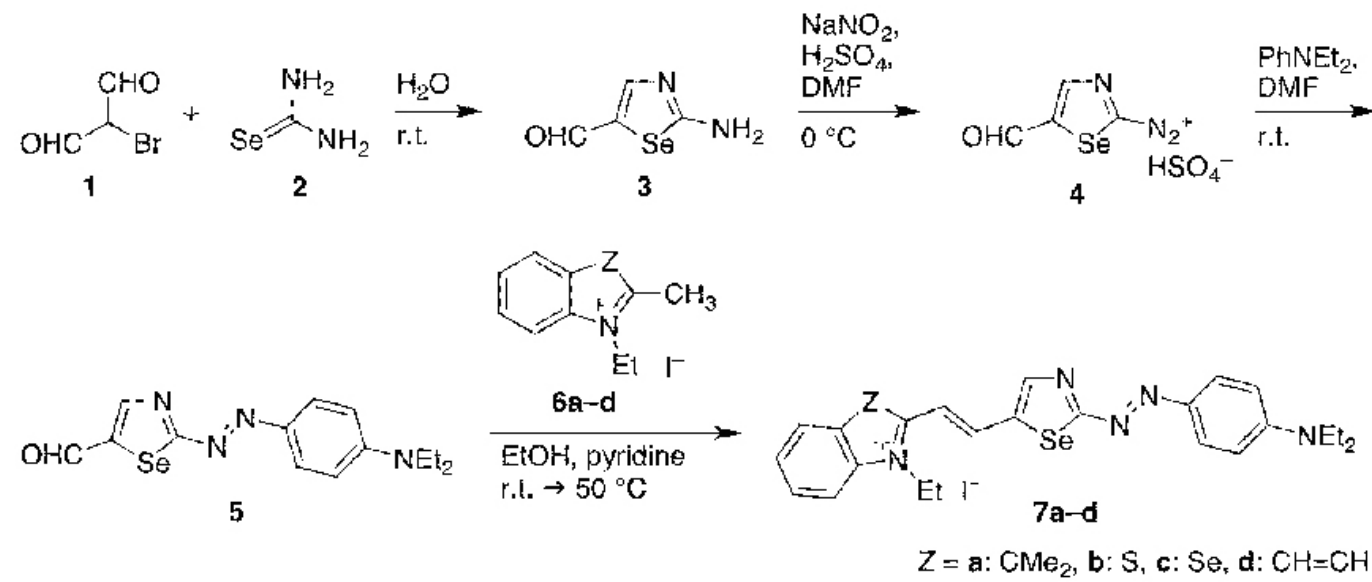

\section{Scheme 1}

Finally, condensation of $\mathbf{5}$ with a quaternary heterocyclic salt $\mathbf{6 a}-\mathbf{d}$ in the presence of pyridine furnished the delocalized cationic azo dyes 7a-d in poor to moderate yields.

All synthesized dyes display a strong absorption $\left(\varepsilon>10^{4} \mathrm{~cm}^{-1} \mathrm{M}^{-1}\right)$ within the range of 684$744 \mathrm{~nm}$ (Table 1); the molar absorption coefficients are approximately 10 times smaller than those of the corresponding thiazole analogs. When compared to the latter, ${ }^{2 \mathrm{~g}}$ the maximum wavelength of absorption of the selenazole-derived dyes is bathochromically shifted from 9 to 30 $\mathrm{nm}$, except in the case of dye $\mathbf{7 b}$, for which a hypsochromic shift of $6 \mathrm{~nm}$ was observed. The largest bathochromic shift was observed for the quinoline-based dye 7d, while the lowest was displayed by dye $7 \mathbf{c}$ derived from benzoselenazole. 
As with their thiazole counterparts, dyes 7a-d show negative solvatochromic behavior on passing from $\mathrm{CH}_{2} \mathrm{Cl}_{2}$ to $\mathrm{DMSO}$, and from this to the even more polar $\mathrm{MeOH}$. ${ }^{9}$ The largest solvatochromic shift was observed for the indolenine dye $7 \mathbf{a}(60 \mathrm{~nm})$.

Table 1. Yields and Vis spectral data for azo dyes 7

\begin{tabular}{ccccc}
\hline Compound & $\begin{array}{c}\text { Yield } \\
(\%)\end{array}$ & $\begin{array}{c}\lambda_{\max }[\mathrm{nm}](\log \varepsilon) \\
\mathrm{CH}_{2} \mathrm{Cl}_{2}\end{array}$ & $\begin{array}{c}\lambda_{\max }[\mathrm{nm}] \\
\text { DMSO }\end{array}$ & $\begin{array}{c}\lambda_{\max }[\mathrm{nm}] \\
\mathrm{MeOH}\end{array}$ \\
\hline 7a & 20 & $744(4.73)$ & 687 & 684 \\
$7 \mathbf{b}$ & 13 & $684(4.76)$ & 648 & 645 \\
$7 \mathbf{c}$ & 17 & $702(4.63)$ & 651 & 645 \\
$7 \mathbf{7 d}$ & 13 & $687(4.36)$ & 642 & 639 \\
\hline
\end{tabular}

In conclusion, a modified Hantzsch method was used to prepare, in rather good yield, 2aminoselenazole-5-carbaldehyde (3), a novel member of the limited family of the biologically important 5-acyl-2-amino-1,3-selenazoles. The Knoevenagel condensation of an azo derivative of 3 with methylene bases generated in situ from benzoazolium and quinolinium salts $\mathbf{6}$ was then used to prepare several new delocalized cationic azo dyes. The so formed dyes display strong absorption around $700 \mathrm{~nm}$, which is, in general, bathochromically shifted in relation to that of the known thiazole analogues.

\section{Experimental Section}

General Procedures. All reagents were of the highest purity available, purchased from SigmaAldrich Company, and were used as received. Bromomalonaldehyde $(\mathbf{1})^{10}$ and the quaternary ammonium salts $6 \mathbf{6}-\mathbf{d}$ were prepared according to the literature. ${ }^{11}$ Reactions were monitored by thin-layer chromatography using $0.25 \mathrm{~mm}$ aluminium-backed silica-gel plates (Merck $60 \mathrm{~F}_{254}$ ). Melting points were measured in open capillary tubes in a Büchi 530 melting point apparatus. IR spectra were recorded on a Mattson 5000 FT IR spectrophotometer. UV/Vis spectra were performed on a Perkin-Elmer Lambda 6 instrument. ${ }^{1} \mathrm{H}$ and ${ }^{13} \mathrm{C}$ NMR spectra were recorded on Bruker ARX 400 or ACP 250 spectrometers. Chemical shifts are reported with respect to the solvent or TMS as internal standard. Fast Atom Bombardment High Resolution mass spectra (FABHRMS) were determined on a Micromass AutoSpec M spectrometer, operating at $70 \mathrm{eV}$, using a matrix of 3-nitrobenzyl alcohol (3-NBA).

2-Aminoselenazole-5-carbaldehyde (3). To a suspension of bromomalonaldehyde (1) (5.00 g, $33.11 \mathrm{mmol})$ in water $(108 \mathrm{~mL})$, vigorously stirred under $\mathrm{N}_{2}$, was added selenourea (2) (4.16 g, $33.11 \mathrm{mmol})$. The mixture was stirred at room temperature for $24 \mathrm{~h}$, then poured onto ice and neutralized with aqueous ammonia (25\%). The precipitated product was collected by filtration under reduced pressure, washed with cold water and dried under reduced pressure over $\mathrm{P}_{2} \mathrm{O}_{5}$ to 
afford chromatographically pure 3. Upon concentration of the filtrate under reduced pressure, an additional crop of product with same purity could be obtained. Orange-reddish solid (3.08 g, 53\%); mp $183{ }^{\circ} \mathrm{C}$ (dec.). $\mathrm{R}_{f} 0.56(\mathrm{MeOH}) . \mathrm{IR}(\mathrm{KBr}): \tilde{v}_{\max } 3360(\mathrm{w}), 3274(\mathrm{w}), 3065(\mathrm{w}), 2838$ (w), 1613 (s), 1532 (w), 1495 (s), 1396 (m), 1297 (w), 1206 (s), 1042 (w), 789 (w), $644 \mathrm{~cm}^{-1}$ (w). ${ }^{1} \mathrm{H}$ NMR (400.13 MHz, $\mathrm{CDCl}_{3}$ ): $\delta 9.59$ (s, $\left.1 \mathrm{H}, \mathrm{CHO}\right), 8.55$ (br s, $2 \mathrm{H}, \mathrm{NH}_{2}$, exchanging with $\left.\mathrm{D}_{2} \mathrm{O}\right), 7.89$ (s, 1H, H-4). ${ }^{13} \mathrm{C}$ NMR (100.61 MHz, $\left.\mathrm{CDCl}_{3}\right): \delta 131.4$ (C-5), 156.3 (C-4), 177.2 (C2), 182.8 (CHO). FABHRMS (3-NBA): Calcd. for $\mathrm{C}_{4} \mathrm{H}_{5} \mathrm{~N}_{2} \mathrm{O}^{80} \mathrm{Se}$ : 174.9575; found: 174.9575 . Calcd for $\mathrm{C}_{4} \mathrm{H}_{5} \mathrm{~N}_{2} \mathrm{O}^{80} \mathrm{Se}[\mathrm{M}+\mathrm{H}]^{+}: 176.9567$; found: 176.9565 .

2-(4-Diethylaminophenylazo)selenazole-5-carbaldehyde (5). To a solution of 2-aminoselenazole-5-carbaldehyde (3) (1.5 g, $8.6 \mathrm{mmol})$ in DMF $(28.5 \mathrm{~mL})$ were added concentrated $\mathrm{H}_{2} \mathrm{SO}_{4}$ $(2.8 \mathrm{~mL})$ and $\mathrm{NaNO}_{2}(591 \mathrm{mg}, 8.6 \mathrm{mmol})$, and the mixture was stirred at $0{ }^{\circ} \mathrm{C}$ for about $1 \mathrm{~h}$. To the resulting diazonium salt solution was gradually added a solution of $N, N$-diethylaniline (1.4 $\mathrm{mL}, 8.6 \mathrm{mmol})$ in DMF $(85.5 \mathrm{~mL})$, and the mixture was stirred at r.t. for $1 \mathrm{~h}$. The mixture was then poured into water and thoroughly extracted with $\mathrm{CHCl}_{3}$. The organic extracts were combined, washed sequentially with $1 \%$ aqueous $\mathrm{NaHCO}_{3}$ and water, and dried over anhydrous $\mathrm{Na}_{2} \mathrm{SO}_{4}$. Removal of the solvent at reduced pressure yielded a residue, which was purified by column chromatography (silica gel, petroleum ether/ $\mathrm{Et}_{2} \mathrm{O} 9: 1 \rightarrow 1: 1$ ) and recrystallized from $\mathrm{Et}_{2} \mathrm{O} / \mathrm{CHCl}_{3} / n$-hexane. Purple crystals (491 mg, $\left.17 \%\right)$; mp $136-137{ }^{\circ} \mathrm{C} . \mathrm{R}_{f} 0.68\left(\mathrm{CH}_{2} \mathrm{Cl}_{2} / \mathrm{MeOH}\right.$ 95:5). Vis $\left(\mathrm{CH}_{2} \mathrm{Cl}_{2}\right): \lambda_{\max }(\log \varepsilon): 561(4.58) \mathrm{nm}$. IR (KBr): $\tilde{v}_{\max } 2973(\mathrm{w}), 2919(\mathrm{w}), 1657(\mathrm{~m})$, $1600(\mathrm{~s}), 1543(\mathrm{w}), 1513(\mathrm{w}), 1448(\mathrm{w}), 1414(\mathrm{w}), 1326(\mathrm{~m}), 1304(\mathrm{~s}), 1260(\mathrm{~m}), 1219(\mathrm{~m}), 1120$ (s), $1072(\mathrm{~m}), 1006(\mathrm{~m}), 882(\mathrm{w}), 828(\mathrm{w}), 715 \mathrm{~cm}^{-1}$ (w). ${ }^{1} \mathrm{H}$ NMR $\left(250.13 \mathrm{MHz}, \mathrm{CDCl}_{3}\right) \delta 1.24$ (t, 6H, $J=7.1 \mathrm{~Hz}, \mathrm{CH}_{3}$ ), 3.48 (q, 4H, $J=7.1 \mathrm{~Hz}, \mathrm{CH}_{2}$ ), 6.66, 6.70 (AA', 2H, ArH), 7.87, 7.91 $\left(\mathrm{BB}^{\prime}, 2 \mathrm{H}, \mathrm{ArH}\right), 8.47(\mathrm{~s}, 1 \mathrm{H},=\mathrm{CHN}), 9.82(\mathrm{~s}, 1 \mathrm{H}, \mathrm{CHO})$. FABHRMS (3-NBA): Calcd for $\mathrm{C}_{14} \mathrm{H}_{17} \mathrm{~N}_{4} \mathrm{O}^{78} \mathrm{Se}$ : 335.0575; found: 335.0564. Calcd. for $\mathrm{C}_{14} \mathrm{H}_{17} \mathrm{~N}_{4} \mathrm{O}^{80} \mathrm{Se}[\mathrm{M}+\mathrm{H}]^{+}$: 337.0568 ; found: 337.0565 .

\section{Synthesis of dyes 7a-d. General procedure}

A solution of a quaternary ammonium salt $6(1.0 \mathrm{mmol})$ and the azo dye $5(0.34 \mathrm{~g}, 1.0 \mathrm{mmol})$ in EtOH (ca $15 \mathrm{~mL})$ containing pyridine $(1.5 \mathrm{~mL})$ was stirred between r.t. and $40-50{ }^{\circ} \mathrm{C}$ until complete consumption of the starting azo dye $(6-46 \mathrm{~h})$. The temperature was carefully controlled to avoid the decomposition of both the starting material and the reaction product. The reaction mixture was then cooled, and $\mathrm{Et}_{2} \mathrm{O}$ was added. The resulting dark-blue solid was collected by filtration under reduced pressure, washed several times with $\mathrm{Et}_{2} \mathrm{O}$, dissolved in $\mathrm{CHCl}_{3}$ and the solution washed with water. The organic layer, after being separated by decantation, was dried over anhydrous $\mathrm{Na}_{2} \mathrm{SO}_{4}$ and evaporated to dryness. The resulting residue was recrystallized from $\mathrm{CHCl}_{3} / \mathrm{MeOH} / \mathrm{Et}_{2} \mathrm{O}$ until a chromatographically pure material was obtained (1-3 times).

2-\{2-[2-(4-Diethylaminophenylazo)selenazol-5-yl]vinyl\}-1-ethyl-3,3-dimethyl-3H-indolium iodide (7a). Dark-green solid. (38 mg, 20\%); mp $184{ }^{\circ} \mathrm{C}$ (dec.). $\mathrm{R}_{f} 0.51\left(\mathrm{CH}_{2} \mathrm{Cl}_{2} / \mathrm{MeOH} 9: 1\right)$. Vis $\left(\mathrm{CH}_{2} \mathrm{Cl}_{2}\right): \lambda_{\max }(\log \varepsilon) 744$ (4.73). IR (KBr): $\tilde{v}_{\max } 2971(\mathrm{w}), 1598(\mathrm{~m}), 1572(\mathrm{~s}), 1534(\mathrm{~m}), 1463$ (w), 1242 (s), 1206 (m), 1164 (w), 1108 (s), 1067 (s), 1040 (m), 1004 (w), 940 (w), 879 (w), 768 
$\mathrm{cm}^{-1}(\mathrm{w}) .{ }^{1} \mathrm{H}$ NMR $\left(400.13 \mathrm{MHz}, \mathrm{DMSO}-d_{6}\right): \delta 1.22\left(\mathrm{t}, 6 \mathrm{H}, J=6.9 \mathrm{~Hz}, \mathrm{CH}_{3}\right), 1.42(\mathrm{t}, 3 \mathrm{H}, J=7.1$ $\mathrm{Hz}, \mathrm{CH}_{3}$ ), 1.79 (s, $6 \mathrm{H}, \mathrm{CH}_{3}$ ), 3.63 (br q, $4 \mathrm{H}, J=6.9 \mathrm{~Hz}, \mathrm{CH}_{2}$ ), 4.63 (br q, $2 \mathrm{H}, J=7.1 \mathrm{~Hz}, \mathrm{CH}_{2}$ ), 7.02, 7.04 (AA', 2H, ArH), $7.17(\mathrm{~d}, 1 \mathrm{H}, J=15.4 \mathrm{~Hz},=\mathrm{CHC}), 7.59-7.64(\mathrm{~m}, 2 \mathrm{H}, \mathrm{ArH}), 7.83-$ $7.88(\mathrm{~m}, 4 \mathrm{H}, \mathrm{ArH}), 8.70(\mathrm{~d}, 1 \mathrm{H}, J=15.4 \mathrm{~Hz},=\mathrm{CHC}), 8.79(\mathrm{~s}, 1 \mathrm{H},=\mathrm{CHN}) .{ }^{13} \mathrm{C}$ NMR $(100.61$ MHz, DMSO-d $\left.d_{6}\right): \delta 12.7\left(\mathrm{CH}_{3}\right), 13.6\left(\mathrm{CH}_{3}\right), 25.6\left(\mathrm{C}_{\left.\left(\mathrm{CH}_{3}\right)_{2}\right),} 41.7\left(\mathrm{C}_{\left.\left(\mathrm{CH}_{3}\right)_{2}\right)}\right), 45.2\left(\mathrm{CH}_{2}\right), 51.9\right.$ $\left(\mathrm{CH}_{2}\right), 113.2,114.7,123.0,129.0,129.1,139.0,140.5,142.8,143.7,147.0,153.9,156.7,179.8$, 190.4. FABHRMS (3-NBA): Calcd. for $\mathrm{C}_{27} \mathrm{H}_{32} \mathrm{~N}_{5}{ }^{78} \mathrm{Se}$ : 504.1831; found: 504.1853 . Calcd. for $\mathrm{C}_{27} \mathrm{H}_{32} \mathrm{~N}_{5}{ }^{80} \mathrm{Se}[\mathrm{M}]^{+}: 506.1823$; found: 506.1836.

2-[2-[2-(4-Diethylaminophenylazo)selenazol-5-yl]vinyl]-3-ethylbenzothiazol-3-ium iodide (7b). Dark-green solid (126 mg, 20\%); mp $173{ }^{\circ} \mathrm{C}$ (dec.). $\mathrm{R}_{f} 0.46\left(\mathrm{CH}_{2} \mathrm{Cl}_{2} / \mathrm{MeOH} 9: 1\right)$. Vis $\left(\mathrm{CH}_{2} \mathrm{Cl}_{2}\right): \lambda_{\max }(\log \varepsilon) 684$ (4.76). IR (KBr): $\tilde{v}_{\max } 2970(\mathrm{w}), 1592(\mathrm{~m}), 1574(\mathrm{~m}), 1537(\mathrm{w}), 1443$ (w), 1414 (w), 1253 (s), 1206 (m), 1114 (s), 1069 (s), 1034 (w), 1005 (w), 826 (w), $784 \mathrm{~cm}^{-1}$ (w). ${ }^{1} \mathrm{H}$ NMR (400.13 MHz, DMSO- $\left.d_{6}, 40{ }^{\circ} \mathrm{C}\right): \delta 1.22\left(\mathrm{t}, 6 \mathrm{H}, J=7.1 \mathrm{~Hz}, \mathrm{CH}_{3}\right), 1.47$ (t, $3 \mathrm{H}, J=7.1$ $\mathrm{Hz}, \mathrm{CH}_{3}$ ), 3.61 (br q, $4 \mathrm{H}, J=7.1 \mathrm{~Hz}, \mathrm{CH}_{2}$ ), 4.91 (br q, $2 \mathrm{H}, J=7.1 \mathrm{~Hz}, \mathrm{CH}_{2}$ ), 6.97, 6.99 (AA', $2 \mathrm{H}, \mathrm{ArH}), 7.61(\mathrm{~d}, 1 \mathrm{H}, J=15.2 \mathrm{~Hz},=\mathrm{CHC}), 7.76-7.88(\mathrm{~m}, 4 \mathrm{H}, \mathrm{ArH}), 8.25(\mathrm{~d}, 1 \mathrm{H}, J=8.2 \mathrm{~Hz}$, ArH), 8.42-8.49 (m, 2H, ArH and $=\mathrm{CHC}), 8.57(\mathrm{~s}, 1 \mathrm{H},=\mathrm{CHN}) .{ }^{13} \mathrm{C}$ NMR $(100.61 \mathrm{MHz}$, DMSO- $\left.d_{6}\right): \delta 12.3\left(\mathrm{CH}_{3}\right), 13.8\left(\mathrm{CH}_{3}\right), 44.1\left(\mathrm{CH}_{2}\right), 44.7\left(\mathrm{CH}_{2}\right), 112.6,114.1,116.2,124.1,128.0$, 129.3, 137.9, 140.6, 142.0, 153.2, 188.6. FABHRMS (3-NBA): Calcd. for $\mathrm{C}_{24} \mathrm{H}_{26} \mathrm{~N}_{5} \mathrm{~S}^{78} \mathrm{Se}$ : 494.1082; found: 494.1080. Calcd. for $\mathrm{C}_{24} \mathrm{H}_{26} \mathrm{~N}_{5} \mathrm{~S}^{80} \mathrm{Se}[\mathrm{M}]^{+}:$496.1074; found: 496.1076.

2-[2-[2-(4-Diethylaminophenylazo)selenazol-5-yl]vinyl]-3-ethylbenzoselenazol-3-ium iodide (7c). Dark-brownish solid (114 mg, 17\%); mp $209{ }^{\circ} \mathrm{C}$ (dec.). $\mathrm{R}_{f} 0.51\left(\mathrm{CH}_{2} \mathrm{Cl}_{2} / \mathrm{MeOH} 9: 1\right)$. Vis $\left(\mathrm{CH}_{2} \mathrm{Cl}_{2}\right): \lambda_{\max }(\log \varepsilon) 702$ (4.63). IR (KBr): $\tilde{v}_{\max } 2971(\mathrm{w}), 2924(\mathrm{w}), 1600(\mathrm{~m}), 1568(\mathrm{~s}), 1538$ (m), 1442 (w), 1416 (w), 1379 (w), 1247 (s), 1198 (m), 1113 (s), 1072 (s), 1006 (m), 880 (w), $775 \mathrm{~cm}^{-1}(\mathrm{w}) .{ }^{1} \mathrm{H}$ NMR (400.13 MHz, DMSO-d $\left.d_{6}\right) \delta 1.21$ (t, $\left.6 \mathrm{H}, J=6.8 \mathrm{~Hz}, \mathrm{CH}_{3}\right), 1.43$ (t, $3 \mathrm{H}, J$ $=7.1 \mathrm{~Hz}, \mathrm{CH}_{3}$ ), 3.59 (br q, $4 \mathrm{H}, J=6.8 \mathrm{~Hz}, \mathrm{CH}_{2}$ ), 4.88 (br q, $2 \mathrm{H}, J=7.1 \mathrm{~Hz}, \mathrm{CH}_{2}$ ), 6.95, 6.98 (AA', 2H, ArH), 7.59 (d, $1 \mathrm{H}, J=14.9 \mathrm{~Hz},=\mathrm{CHC}), 7.68(\mathrm{t}, 1 \mathrm{H}, J=8.0 \mathrm{~Hz}, \operatorname{Ar} H), 7.79-7.81(\mathrm{~m}$, $3 \mathrm{H}, \operatorname{Ar} H), 8.21(\mathrm{~d}, 1 \mathrm{H}, J=8.0 \mathrm{~Hz}, \operatorname{ArH}), 8.44(\mathrm{~d}, 1 \mathrm{H}, J=8.0 \mathrm{~Hz}, \operatorname{ArH}), 8.54-8.58(\mathrm{~m}, 2 \mathrm{H}$, $=\mathrm{CHC}$ and $=\mathrm{CHN}) .{ }^{13} \mathrm{C}$ NMR $\left(100.61 \mathrm{MHz}, \mathrm{DMSO}-d_{6}\right): \delta 12.6\left(\mathrm{CH}_{3}\right), 14.1\left(\mathrm{CH}_{3}\right), 45.0\left(\mathrm{CH}_{2}\right)$, 45.2 $\left(\mathrm{CH}_{2}\right), 112.9,117.2,117.9,127.3,127.9,129.0,130.1,138.5,142.1,142.3,143.9,153.4$, 153.7, 179.2, 188.8. FABHRMS (3-NBA): Calcd. for $\mathrm{C}_{24} \mathrm{H}_{26} \mathrm{~N}_{5}{ }^{78} \mathrm{Se}_{2}$ : 540.0534; found: 540.0538. Calcd. for $\mathrm{C}_{24} \mathrm{H}_{26} \mathrm{~N}_{5}{ }^{78} \mathrm{Se}^{80} \mathrm{Se}$ : 542.0526; found: 542.0515. Calcd. for $\mathrm{C}_{24} \mathrm{H}_{26} \mathrm{~N}_{5}{ }^{78} \mathrm{Se}{ }^{80} \mathrm{Se}$ : 542.0526; found: 542.0515. Calcd for $\mathrm{C}_{24} \mathrm{H}_{26} \mathrm{~N}_{5}{ }^{80} \mathrm{Se}_{2}[\mathrm{M}]^{+}$: 544.0519; found: 544.0536.

2-[2-[2-(4-Diethylaminophenylazo)selenazol-5-yl]vinyl]-1-ethylquinolinium iodide (7d). Dark-green solid (80 mg, $13 \%$ ); mp $193{ }^{\circ} \mathrm{C}$ (dec.). $\mathrm{R}_{f} 0.44\left(\mathrm{CH}_{2} \mathrm{Cl}_{2} / \mathrm{MeOH}\right.$ 9:1). Vis $\left(\mathrm{CH}_{2} \mathrm{Cl}_{2}\right)$ : $\lambda_{\max }(\log \varepsilon) 687$ (4.36). IR (KBr): $\tilde{v}_{\max } 2971$ (w), $1584(\mathrm{~s}), 1502(\mathrm{w}), 1410(\mathrm{w}), 1329(\mathrm{w}), 1256$ (m), 1206 (m), 1115 (s), 1072 (m), 1006 (w), 826 (w), $759 \mathrm{~cm}^{-1}$ (w). ${ }^{1} \mathrm{H}$ NMR (400.13 MHz, DMSO- $d_{6}$ ): $\delta 1.20$ (t, $6 \mathrm{H}, J=7.0 \mathrm{~Hz}, \mathrm{CH}_{3}$ ), 1.55 (t, $3 \mathrm{H}, J=7.0 \mathrm{~Hz}, \mathrm{CH}_{3}$ ), 3.56 (br q, $4 \mathrm{H}, J=7.0$ $\mathrm{Hz}, \mathrm{CH}_{2}$ ), 5.09 (br q, $2 \mathrm{H}, J=7.0 \mathrm{~Hz}, \mathrm{CH}_{2}$ ), 6.91, 6.93 (AA', 2H, $\mathrm{Ar} H$ ), 7.40 (d, $1 \mathrm{H}, J=15.0 \mathrm{~Hz}$, $=\mathrm{CHC}), 7.76,7.78\left(\mathrm{BB}^{\prime}, 2 \mathrm{H}, \mathrm{ArH}\right), 7.92(\mathrm{t}, 1 \mathrm{H}, J=7.5 \mathrm{~Hz}, \mathrm{ArH}), 8.16$ (t, $\left.1 \mathrm{H}, J=7.5 \mathrm{~Hz}, \operatorname{ArH}\right)$, 
$8.32(\mathrm{~d}, 1 \mathrm{H}, J=7.5 \mathrm{~Hz}, \mathrm{ArH}), 8.40(\mathrm{~s}, 1 \mathrm{H},=\mathrm{CHN}), 8.53-8.56(\mathrm{~m}, 3 \mathrm{H}, \mathrm{ArH}$ and $=\mathrm{CHC}), 9.00(\mathrm{~d}$, $1 \mathrm{H}, J=8.9 \mathrm{~Hz}, \mathrm{ArH}) .{ }^{13} \mathrm{C} \mathrm{NMR}\left(100.61 \mathrm{MHz}, \mathrm{DMSO}-d_{6}\right): \delta 12.5\left(\mathrm{CH}_{3}\right), 14.0\left(\mathrm{CH}_{3}\right), 44.8$ $\left(\mathrm{CH}_{2}\right), 46.4\left(\mathrm{CH}_{2}\right), 112.6,118.8,119.9,120.8,128.0,128.8,130.2,135.0,138.0,139.3,141.0$, 142.0, 143.7, 151.7, 153.0, 154.1, 187.4. FABHRMS (3-NBA): Calcd. for $\mathrm{C}_{26} \mathrm{H}_{28} \mathrm{~N}_{5}{ }^{78} \mathrm{Se}$ : 488.1518; found: 488.1519. Calcd. for $\mathrm{C}_{26} \mathrm{H}_{28} \mathrm{~N}_{5}{ }^{80} \mathrm{Se}[\mathrm{M}]^{+}$: 490.1510; found: 490.1508 .

\section{Acknowledgements}

Fundação para a Ciência e a Tecnologia (Portugal), POCI 2010 and FEDER are gratefully acknowledged for the funding of the Project "Azo Dyes for Photodynamic Therapy" (POCI/QUI/57913/2004).

\section{References}

1. Konstantinou, I. K.; Albanis, T. A. Appl. Catal. B: Environ. 2004, 49, 1.

2. (a) Bello, K. A.; Griffiths, J. Chem. Commun. 1986, 1639. (b) Hall, N.; Patel, P. GB. Patent 2237 804, 1991. (c) Gregory, P. Eur. Patent 280 434, 1988. (d) Yasui, S.; Matsuoka, M.; Takao, M.; Kitao, T. J. Soc. Dyers Colour 1988, 104, 284. (e) Dickey, J. B.; Towne, E. B.; Bloom, M. S.; Moore, W. H.; Hill, H. M.; Heynemann, H.; Hedberg, D. G.; Sievers, D. C.; Otis, M. V. J. Org. Chem. 1959, 24, 187. (f) Griffiths, J.; Lee, W. J. Adv. Colour Sci. Technol. 2002, 5, 99. (g) Salvador, M. A.; Reis, L. V.; Almeida, P.; Santos, P. F. Tetrahedron 2008, 64, 299. (h) Salvador, M. A.; Reis, L. V.; Almeida, P.; Santos, P. F. Dyes and Pigments, in print.

3. Dickey, J. B.; Towne, E. B.; Bloom, M. S.; Moore, W. H.; Hill, H. M.; Heynemann, H.; Hedberg, D. G.; Sievers, D. C.; Otis, M. V. J. Org. Chem. 1959, 24, 187.

4. Zollinger, H. Color Chemistry - Synthesis, Properties and Applications of Organic Dyes and Pigments; VCH: Weinheim, 1991; p 146.

5. Keil, D.; Flaig, R.; Schroeder, A.; Hartmann, H. Dyes and Pigments 2001, 50, 67.

6. (a) Frishberg, M. D. U.S. Patent 4,225,719, 1980. (b) Asato, G.; Berkelhammer, G.; Moon, E. L. J. Med. Chem. 1969, 12, 374. (c) Dorlars, A. Ger. Patent 1,182,234, 1964.

7. Koketsu, M.; Ishihara, H. Curr. Org. Chem. 2003, 7, 175; and references cited therein.

8. (a) Liebscher, J.; Hartmann, H. Z. Chem. 1976, 16, 18. (b) Kantlehner, W.; Haubner. M.; Vettel, M. J. Prakt. Chem. 1996, 338, 403. (c) Koketsu, M.; Ando, H.; Ishihara, H. Synthesis 2006, 1,31 .

9. (a) Reichardt, C. Solvents and Solvent Effects in Organic Chemistry; Wiley-VCH: Weinheim, 2003; pp 418-424. (b) ibid. pp 472-475.

10. Trofimenko, S. J. Org. Chem. 1963, 28, 3243.

11. Pardal, A. C.; Ramos, S. S.; Santos, P. F.; Reis, L. V.; Almeida, P. Molecules 2002, 7, 320. 